\title{
Rats show preference for delayed rewards on the radial maze
}

\author{
Miranda C. Feeney and William A. Roberts \\ University of Western Ontario, London, Ontario, Canada
}

\begin{abstract}
Rats on an eight-arm radial maze chose between four arms on which a small reward could be obtained after a short delay and four arms on which a larger reward could be obtained after a longer delay. Experiments 1 and 2 showed that rats preferred the long-delay, large-reward arms over the short-delay, small-reward arms. This preference was particularly marked when the arms were made into enclosed alleys. Experiment 3 showed that this effect was not produced by a preference for staying in enclosed alleys. We argue that the rats endured longer delays to obtain larger rewards when fear of predation was minimized.
\end{abstract}

It has long been known that two reinforcement variables, delay and amount, have a major impact on learning and operant responding. In a classic illustration of the effect of delay of reinforcement (Grice, 1948), rats were exposed to black and white stimuli associated with food or no food in a two-choice discrimination runway box. The delay between the time a rat entered the goal box and the time the reward was delivered varied between 0 and $10 \mathrm{sec}$. The learning curves indicated a steep delay-ofreinforcement gradient; as the delay increased, it became increasingly difficult for rats to learn the discrimination, with most rats failing to learn it at the 10 -sec delay. On the basis of this experiment and others, it became accepted that little learning occurs with an extended delay of reinforcement (but see Lett, 1973, and Lieberman, McIntosh, \& Thomas, 1979).

In the case of amount of reinforcement, it has been observed repeatedly that animals will respond faster and at a higher rate for a large reinforcer than for a small one. Thus, rats will run down a runway or press a bar faster for a larger amount of food than they will for a smaller amount (Meltzer \& Brahlek, 1968; Roberts, 1969). Given a choice between responses leading to small or large rewards, animals choose the large reward (Clayton, 1964; Davenport, 1962). Although it was once thought that the amount of reinforcement directly affected associative strength (Hull, 1943), a more contemporary point of view is that the amount of reinforcement produces differences in incentive motivation (Spence, 1956).

Given the strong influence of the delay and amount variables on operant behavior, it is interesting to observe what happens when these variables are pitted against one another. In the self-control paradigm, subjects are given a choice between an immediate, smaller reward and a larger, delayed reward. For example, rats and pigeons have been offered a choice between a response that leads to $2 \mathrm{sec}$ of reward after a 0.1 -sec delay and another response that leads to $6 \mathrm{sec}$ of reward after a 6-sec delay. Choice of the 6-sec delayed reward has been defined as self-control, and choice of the more immediate 2-sec reward has been defined as impulsiveness. Given conditions set at values within this range of delay lengths, animals typically prefer the immediate reward and thus show impulsiveness (Logue, 1988; Rachlin $\&$ Green, 1972). Choice of the self-control alternative has been found only under unusual training conditions, such as (1) first establishing a preference for the larger reward at a common long delay and then gradually fading in the shorter delay (Mazur \& Logue, 1978); (2) adding a constant time to both delays, thus making the choice between, for example, a small reward after a 4.1-sec delay and a large reward after a 10-sec delay (Green \& Estle, 2003); (3) preexposing animals to the delay conditions of the choice alternatives (Eisenberger, Masterson, \& Lowman, 1982); and (4) manipulating the effort involved in making choices (Chelonis, Logue, Sheehy, \& Mao, 1998; Eisenberger, Weier, Masterson, \& Theis, 1989).

In contrast, when human subjects are tested with the basic self-control paradigm, they show self-control by preferring the large delayed reward over the small immediate reward (King \& Logue, 1987; Logue, Peña-Correal, Rodriguez, \& Kabela, 1986). However, most of the research on humans has been conducted with monetary or token rewards. When foods are used as the rewards, human self-control is affected. The results of several studies have indicated a variety of effects, including these: (1) Adults show less self-control in situations in which they are confronted with a choice between food rewards delivered more immediately throughout a session and monetary rewards delivered at the end of a session than in situations in which points are exchanged for both money and food at the end of sessions (Forzano \& Logue, 1994). (2) Adults show more self-control for food rewards when they have

W.A. Roberts, roberts@uwo.ca 
already been fed than when they have not been fed already (Kirk \& Logue, 1997). (3) Choices made by preschool children can be influenced by visual food cues, with more self-control observed when the cues are absent than when they are present (Forzano \& Logue, 1995). These results suggest that food may be a particularly difficult reward to resist, for both animals and humans.

One explanation for favoring a short delay to reward is that animals have a very short time window for future events. If a rat cannot anticipate an outcome $6 \mathrm{sec}$ into the future, its choice in a self-control experiment may be between the immediate small reward and nothing (Logue, 1988). Other studies of the time horizon in rats suggest, however, that rats can anticipate future changes in reinforcers as far as 15-30 min into the future. For instance, rats pressed a bar for a modest reinforcer at a slower rate when a richer reinforcer was going to be delivered $15 \mathrm{~min}$ later than when no change in the reinforcer would occur in the future (Flaherty \& Checke, 1982). A second account for the predominance of impulsive behavior in rats is that the value of a reinforcer becomes discounted over a delay. Thus, $6 \mathrm{sec}$ of access to food after $6 \mathrm{sec}$ may be perceived as less reward than $2 \mathrm{sec}$ of access to food after $0.1 \mathrm{sec}$ (Green \& Meyerson, 2004).

Quantity of food and delays prior to the consumption of food are understood somewhat differently in models of optimal foraging theory. These models suggest that animals have been shaped by evolutionary forces to make choices that tend to maximize the energy obtained per unit of time spent foraging $(\mathrm{E} / \mathrm{T})$ and, ultimately, the genetic fitness of the organism (Stephens \& Krebs, 1986). According to this framework, therefore, animals should prefer response alternatives that lead to both large rewards and short delays to get to reward. Large rewards should be preferred because they provide more energy than small rewards. Short delays should be preferred because they maximize the $\mathrm{E} / \mathrm{T}$ ratio. In addition, short delays mean less time spent foraging. When time must be spent searching for food, there is a risk that another forager may find the food first or that the forager may be attacked by predators (Lima, 1985; Lima, Valone, \& Caraco, 1985). Overall, a forager might prefer an immediate small amount of food to a delayed larger amount of food because the E/T ratio is adequate and the risk is low. In theories of foraging based on natural situations, animals' behavior is indicative of their being programmed to value one aspect of each alternative (the short delay of the smaller, sooner option or the greater amount of food in the larger, later option). Responses likely represent a trade-off that depends on which variable would be weighted more in light of the given conditions.

The radial arm maze has been used to study spatial memory in rats, but this apparatus may also reveal evidence of foraging strategies in rats. A typical maze consists of eight arms that radiate outward from a central platform, a pattern similar to that of the underground burrow systems wild rats excavate (Neider, Cagnin, \& Parisi, 1982; Pisano \& Storer, 1948). When food is placed at the end of each arm, rats tend to visit each arm and consume the food there without revisiting arms, indicating memory for previously visited arms (Olton \& Samuelson, 1976). If larger chunks of food are placed at the ends of the arms, rats show central-place foraging by carrying the food to the maze's center before consuming it (Phelps \& Roberts, 1989; Whishaw \& Tomie, 1989). The center of the maze may be perceived as a safer place to consume food than the end of an arm, since the center offers a greater number of escape routes (Roberts, Phelps, \& Schacter, 1992). When different quantities of food are placed at the ends of the arms, rats learn to discriminate the maze arms according to the amount of reward at the end of each one, and they develop a preference for entering the arms in descending order of those with the greatest to those with the least amounts of food (Hulse \& O'Leary, 1982; Roberts, 1992). These findings suggest that rats negotiating a radial maze go to the greatest sources of energy first and carry larger pieces of food to the safest part of the maze for consumption. These behaviors minimize both the risk of personal predation and the possibility that other foragers might obtain the larger amounts of food before they do.

Given the many parallels between optimal foraging and self-control theories, an integration of paradigms may provide a more comprehensive, naturalistic examination of some of the coexisting factors while also eliminating some of the limitations of typical studies. In fact, animals display foraging patterns that naturally mimic the alternatives available in self-control studies, choosing smaller rewards at closer, more immediately available sites while collecting larger rewards at farther removed, more delayed locations (Jackson, 2001; Lair, Kramer, \& Giraldeau, 1994). It is not clear how animals would approach the amount-delay tradeoff in a more naturalistic foraging situation, such as on the radial maze.

Since delay of reward has not been directly examined on the radial maze, it was studied in the experiments reported here by varying the amount of time a rat had to spend on an arm after the rat had chosen the arm, but before the rat was allowed to enter the goal area at the arm's end. Delay and amount of reward were varied among arms. The purpose of these experiments was to explore the possibility that rats might show increased or reduced sensitivity to delay of reward properties depending on the conditions under which they foraged on the maze.

A preliminary experiment was designed to examine the effect of introducing a delay to reward to rats' choice behavior - specifically, to determine whether rats would discriminate among radial maze arms according to the delay associated with each arm. This experiment is not reported in detail in this article, but the direction taken in the subsequent experiments was informed by the design and results, so a brief report is warranted. On half of the arms, rats experienced only a 1 -sec delay before they were given access to a food reward. Choosing any of the remaining arms meant that the rats experienced a $10-\mathrm{sec}$ delay before they were given access to a reward. Reward magnitude after both delays was equal in the first phase of the experiment.

In subsequent phases of the experiment, the magnitude of the rewards associated with the long-delay arms increased. Rats have been shown to be sensitive to reward magnitude on the radial maze (see, e.g., Hulse \& O'Leary, 1982; Roberts, 1992), but the combined effects of delay and reward have 
not been studied. In Phase 1, in which both the short (1-sec) and the long (10-sec) delay conditions delivered one raisin, rats learned to enter short-delay arms before entering longdelay arms. During Phases 2, 3, and 4, in which the number of raisins provided on the long-delay arms increased to two, three, and five, respectively, a similar but clearer picture of the pattern of responding was observed. Over these final three phases, the rats' behavior showed minimal variability, and the strong tendency to enter short-delay arms before entering long-delay arms was maintained despite increases in the amount of the larger but more delayed reward alternative. Since there was no difference in reinforcer magnitude in Phase 1, this preference for the short-delay arms is predicted if rats are capable of discriminating the arms according to the associated delays to reward. It can be concluded that the rats learned the associations because the expected preference was exhibited.

One explanation for the lack of an effect of magnitude differences is that the delay was simply too long for the larger reward to ever be considered more valuable, given the amounts used in the study. Davenport (1962) found that rats showed indifference when the small reward was two pellets delayed for $1 \mathrm{sec}$ and the large reward was either eight pellets delayed for 10-30 sec or four pellets delayed for $7 \mathrm{sec}$. Logan (1965) suggested that the indifference point between one and three pellets occurred when the smaller reward was delivered after a 1-sec delay and the larger after a delay of approximately $8-14 \mathrm{sec}$. These findings suggest that rats were at best indifferent to choice alternatives with delays and magnitudes similar to those used in the present study.

Another possibility is that because in the preliminary study only delay to reward was introduced initially, delay became the more salient factor and blocked potential effects of reward magnitude. Rats were exposed to the differential delay parameters for 12 days before reward magnitude differences were introduced; in the course of those days, a strategy of navigating the maze environment based on delay to reward may have developed. That is, the data suggest the possibility that learning about one of the variables in the choice situation, delay to reward, may have blocked learning about the subsequently introduced variable, magnitude of reward.

In Experiments 1 and 2 reported here, different groups of rats were trained with small, immediate rewards on some arms of the maze and with large, delayed rewards on other arms. It was found that under certain conditions of maze foraging, rats were less influenced by delay than by amount and that they preferred the arms with a delayed large reward.

\section{EXPERIMENT 1}

A between-subjects design was used in Experiment 1. Both delay to reward and reward magnitude differences were introduced from the start of testing to eliminate the possibility that initial experience with one variable would block the influence of the second. As well, the delay to the smaller reward (one pellet) was consistent across all groups and lasted $1 \mathrm{sec}$. The delay to the larger reward (five pellets), on the other hand, varied among groups, lasting either 2,5 , or $10 \mathrm{sec}$. In this fashion, it could be determined whether choice patterns in this delay and reward comparison situation would be similar across different delays, or if the integrated delay and amount contingencies would produce different patterns of behavior.

\section{Method}

Animals. The animals were 15 (5 per group) experimentally naive, adult male hooded Long-Evans rats supplied by Charles River Laboratories, Quebec. At the start of testing, the animals were approximately 60 days old and weighed between 385 and $460 \mathrm{~g}$ $(M=391 \mathrm{~g})$. The rats were maintained on a food-restricted diet of 20-25 g of food per day throughout the course of training and testing and had continuous free access to water. Lights in the cage room were kept on a 12:12-h light:dark cycle, with onset at 7:00 am and offset at 7:00 pm.

Apparatus. A raised, open, eight-arm radial maze constructed of $2.5-\mathrm{cm}$ thick plywood was used (see Figure 1). The maze was composed of a central platform and eight arms that radiated from the platform, with adjacent arms separated by the same angular distance. The diameter of the platform was $35.5 \mathrm{~cm}$, and the eight arms each measured $79 \mathrm{~cm}$ long $\times 9 \mathrm{~cm}$ wide. Additionally, sheets of black poster board ( $25 \mathrm{~cm}$ long $\times 22 \mathrm{~cm}$ high) were attached to one side of the arms at the central platform connection to prevent rats from jumping between arms. Two wooden frames standing $30-\mathrm{cm}$ high were installed over each arm, one at the point where the arm connected to the central platform and a second $43.3 \mathrm{~cm}$ down the arm. Each frame held a $15.3 \mathrm{~cm}$ high $\times 10 \mathrm{~cm}$ wide white melamine guillotine door that could be raised and lowered using a piece of fishing line attached to a control board on the testing room wall. When the guillotine doors were raised, the opening available for the rats to pass through measured $9 \mathrm{~cm}$ wide $\times 14 \mathrm{~cm}$ high. A $12.6 \mathrm{~cm} \times 16.6 \mathrm{~cm}$ piece of black poster board was attached to the top half of the frame to prevent rats from climbing over the guillotine doors when they were lowered.

A gray PVC end cap was attached to each arm $4.5 \mathrm{~cm}$ (measured to the middle of the cap) from the distal end. The PVC caps, which were used as food cups, measured $5.5 \mathrm{~cm}$ in diameter and $2 \mathrm{~cm}$ in depth. The maze was supported by nine pieces of wooden dowling, one under the middle of the central platform and one centered under each arm. The dowling legs elevated the maze $61 \mathrm{~cm}$ above the testing room floor. The central platform was painted white and the arms were painted flat black. A single-bulb light fixture that held a $60-\mathrm{W}$ bulb was suspended $60 \mathrm{~cm}$ above the middle of the central platform. The testing apparatus was set up in a room that measured $3.5 \times 3.5 \mathrm{~m}$ and provided ample extramaze cues, including eight wooden boxes, a small table supporting a black and white television, a chair, a wooden lectern, and two doors. In Experiment 1, 45-mg sucrose pellets (Noyes Precision Pellets, Research Diets Inc.) were used as the food reward, which rats always ate at the food cup on the end of an arm.

Procedure. Rats were trained to collect food rewards from the food cups on the radial maze arms and were habituated to the movement of the guillotine doors. For each rat, four randomly selected arms were designated as the short-delay arms, and the remaining four were designated as the long-delay arms. These designated sets were different across rats, but the unique sets remained constant throughout testing for each individual. Rats were then randomly assigned to one of three groups $(n=5)$. For all three groups, the short delay was set at $1 \mathrm{sec}$, and one sucrose pellet was always placed on the short-delay arms. The long delay varied among the three groups, lasting 2, 5, or $10 \mathrm{sec}$. Five sucrose pellets were always placed on the long-delay arms. Each rat was given 1 trail per day, for a total of 30 trials.

\section{Results}

For the arm entries recorded on each trial, the rank order of arm entries was determined for each arm. The 


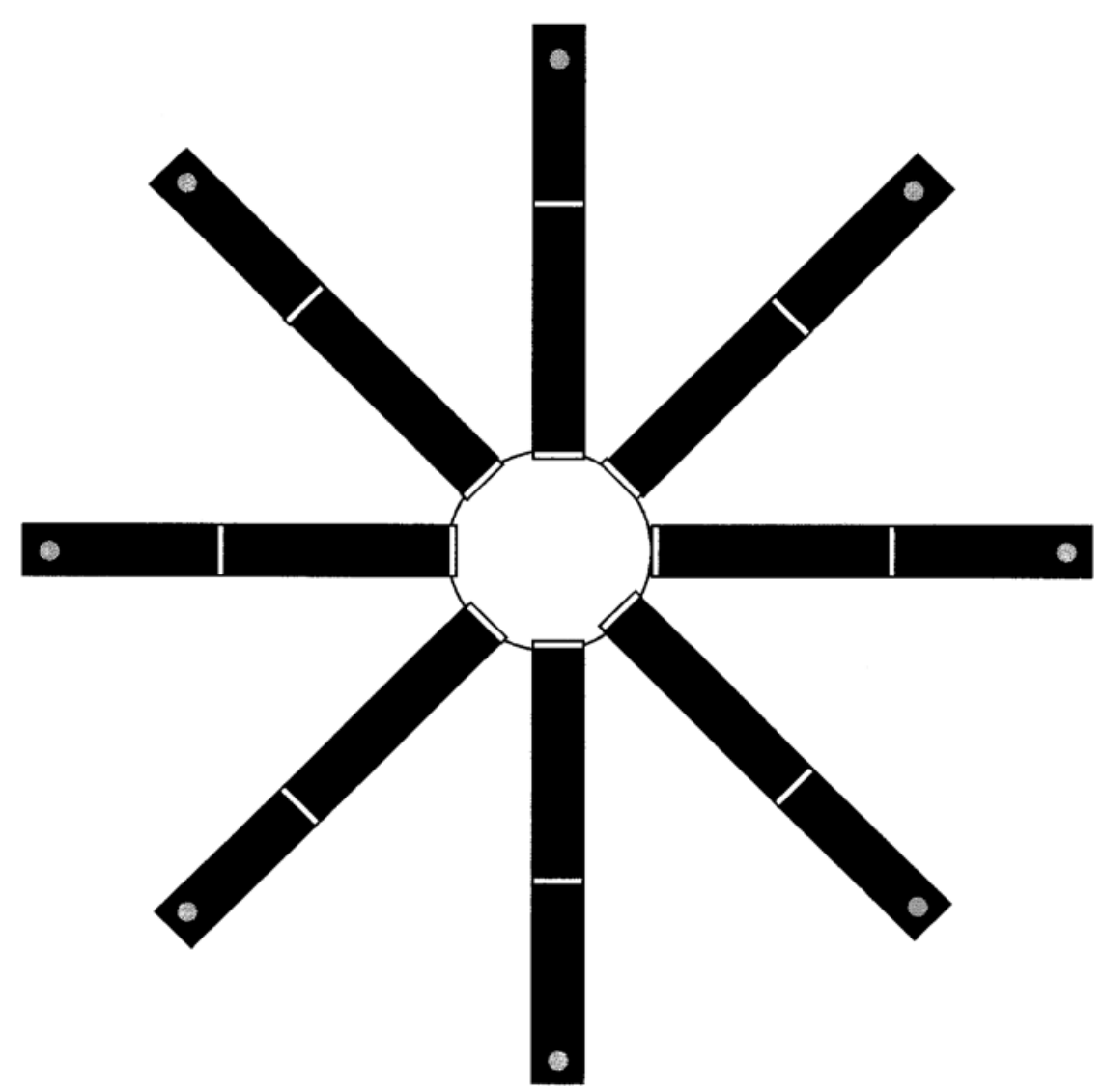

Figure 1. Top view of the radial maze showing the locations of doors that were closed to confine rats on the arms during delays.

means of rank orders of arm entries were calculated for the short- and long-delay arms to yield a mean rank entries (MRE) score for each. The MRE plots for each group during Experiment 1 are shown, in blocks of five trials, in Figure 2. The 2-sec long-delay group (Panel A) showed the earliest (Block 3) and largest separation between the MREs for long- and short-delay arms. The diverging lines clearly demonstrate that over the last four blocks of Experiment 2, the 2-sec long-delay group entered the longdelay arms earlier than they entered the short-delay arms. The separation for the 5-sec long-delay group (Panel B) occurred slightly later in testing (Block 4) and was not as pronounced, though it was in the same direction as that for the 2-sec long-delay group and was maintained until the end of testing. The short- and long-delay MRE plots for the 10-sec long-delay group demonstrate that this group of rats never developed a preference for either delay-reward option, since there is virtually no separation between the MREs for long- and short-delay arms, and the rankings show no development of a consistent pattern.

A difference score was calculated for each rat for each block of five trials by subtracting the long-delay MRE from the short-delay MRE. Figure 3 depicts the mean difference scores for each group across blocks. One-sample $t$ tests were used to compare the difference scores against zero for each group. For the 2-sec long-delay group, the mean difference score $(M=.94, S D=.61)$ was significantly greater than zero $[t(4)=3.48, p=.025]$. For the 5 -sec long-delay group, the mean difference score $(M=$ $.60, S D=.47$ ) also reached significance at a level greater than zero $[t(4)=2.85, p=.046]$. Finally, in the case of the 10-sec long-delay group, the difference score was not significantly different from zero $(M=.07, S D=.33$, $p=.66$ ). A 3 (groups) $\times 6$ (blocks) repeated measures ANOVA was used to compare difference scores across the three delay conditions and the six blocks of five trials each. The interaction of block $\times$ group approached but did not reach statistical significance at the .05 level $[F(10,55)=1.92, p=.06]$. A significant effect of block was observed $[F(5,55)=3.65, p=.01]$. Post hoc pairwise comparisons using the LSD procedure indicated that the difference scores obtained in Blocks 1 and 2 differed from the score obtained in Block 4. No other significant differences between blocks were found. A significant group effect was also observed $[F(2,12)=4.47, p=.04]$. Post hoc tests, again using the LSD procedure, showed that the 2-sec long-delay group differed from the 10-sec longdelay group. No other differences were significant.

Six follow-up one-way ANOVAs were conducted to compare the difference scores among the three groups at each block. No significant effect was observed during Blocks 1,2 , or $3(F \mathrm{~s}<1.0)$. The one-way analysis for 

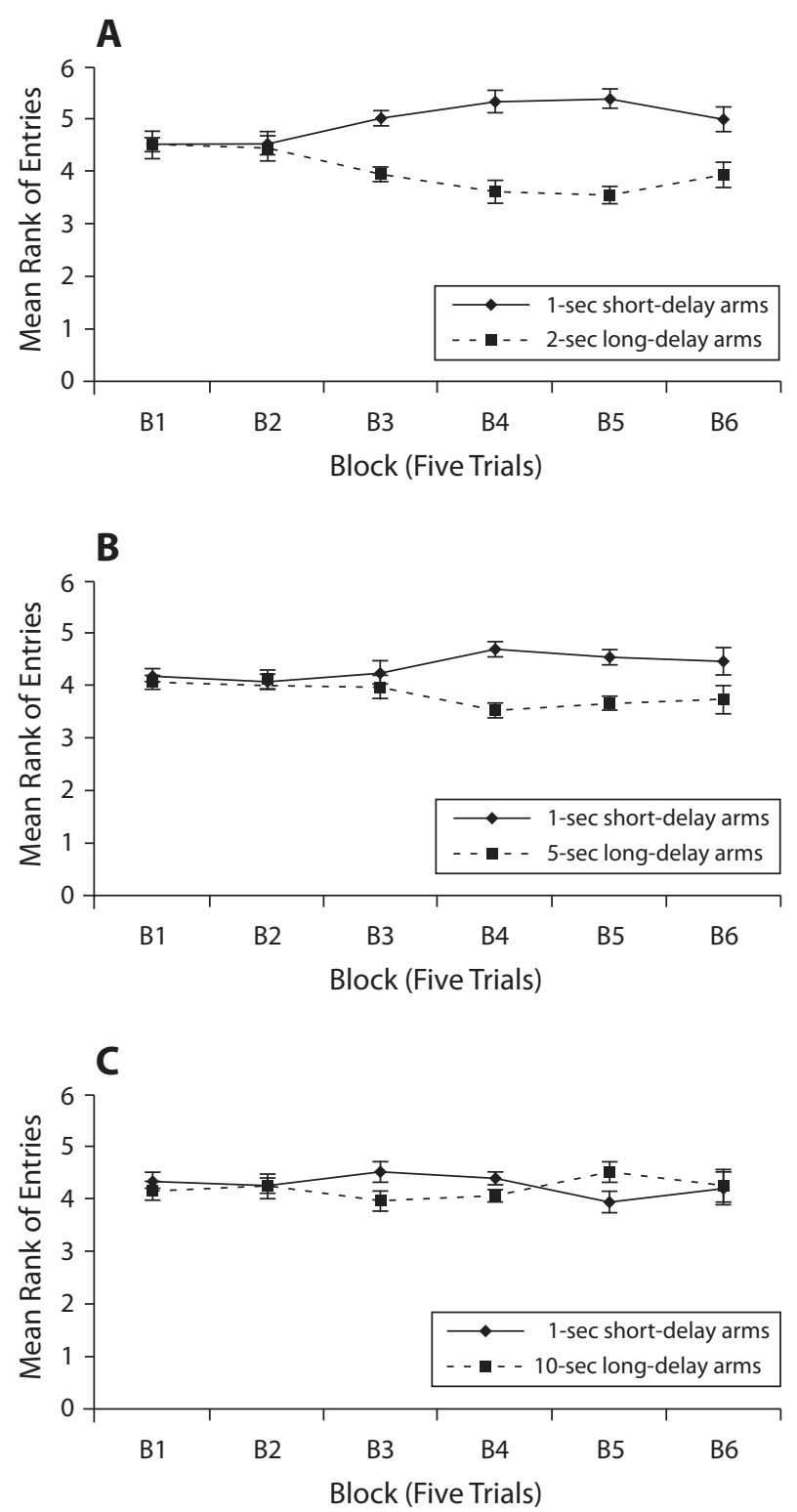

Figure 2. The mean ranks of entries into short- and long-delay arms, calculated for blocks of five trials in Experiment 1. The short delay was always $1 \mathrm{sec}$, but the long delay varied across groups from $2 \mathrm{sec}$ (A) to $5 \mathrm{sec}$ (B) to $10 \mathrm{sec}$ (C). Error bars represent standard errors of the mean.

Block 4 revealed a significant effect of group $[F(2,12)=$ $4.33, p<.05]$. Post hoc comparisons using the LSD procedure indicated that the 2-sec long-delay group had a significantly higher mean difference score than did the 10 -sec long-delay group. No other significant differences were observed. The analysis of Block 5 data also demonstrated a significant effect of group $[F(2,12)=11.55$, $p<.01]$. In this case, the 10 -sec long-delay group had a significantly lower mean difference score than did the 2 -sec and 5-sec long-delay groups. No other significant differences were indicated. Last, the one-way ANOVA for Block 6 failed to demonstrate a significant effect of group $(F<1.0)$.

\section{Discussion}

Rats choosing between one pellet delayed for $1 \mathrm{sec}$ and five pellets delayed for either 2 or $5 \mathrm{sec}$ preferred arms providing the larger but later reward. This effect was shown earliest and most strongly in the 2-sec long-delay group. For the 5-sec long-delay group, the preference was somewhat reduced and consistency was reached later in testing. The 10-sec long-delay group exhibited no preference; rats confronted with a choice between one pellet delayed for $1 \mathrm{sec}$ and five pellets delayed for $10 \mathrm{sec}$ showed indifference to the two choices. Thus, if a preference was shown across rats, it was for the larger, later reward. These results indicate greater control with regard to amount of reward than with regard to delay to reward in this radial maze foraging situation.

One potential explanation for the findings of the present experiment is differential immediate secondary reinforcement. Although responses that are followed by immediate reinforcement become strengthened and repeated, situations involving delays to reward may also produce response acquisition through secondary reinforcement (Spence, 1947). Spence noted that many cases of gradients of response strength and learning based on delay of reward involved delays endured in a compartment next to the box from which the food reward was obtained. It has been hypothesized that the consistent temporal and spatial relationship between the delay compartment and the food box caused differential cues inherent to the delay chambers to acquire reinforcing properties. Because a choice response was followed immediately by entrance into a delay chamber, the properties of the chamber may have reinforced the response (Spence, 1947).

For example, in studying the effect of delay to reward, Grice (1948) made available specific secondary cues for two groups of rats receiving rewards $5 \mathrm{sec}$ after choice responses. For one group, the goal boxes were painted the same color as the black and white alley cues associated with food or no-food outcomes. For the second group, different obstacles for the rats to navigate were placed in the two alleys. Compared with control rats given no secondary cues, who required an average of 580 trials to learn the discrimination, the rats with the black and white goal boxes successfully completed the task in only 155 trials, and those with obstacles met the criterion in 295 trials. Thus, both groups with secondary cues available learned significantly faster than did the control group.

In Experiment 1, every choice led to a food reward, so cues in the delay areas would be associated with food in all cases. The delay areas were not enclosed, which allowed the rats to experience different views of the environment on each arm. Rats in two of the groups developed a preference for one set of arms (i.e., that with the larger rewards) over the other, so it is likely that when the reward was obtained, the spatial locations were retained in memory. Consequently, the different views of the room available in the different arms could have come to be associated with different reward magnitudes. The possible secondary reinforcement from environmental cues may have been stronger for the larger rewards, which may have allowed the rats to more willingly endure the longer delays and in 


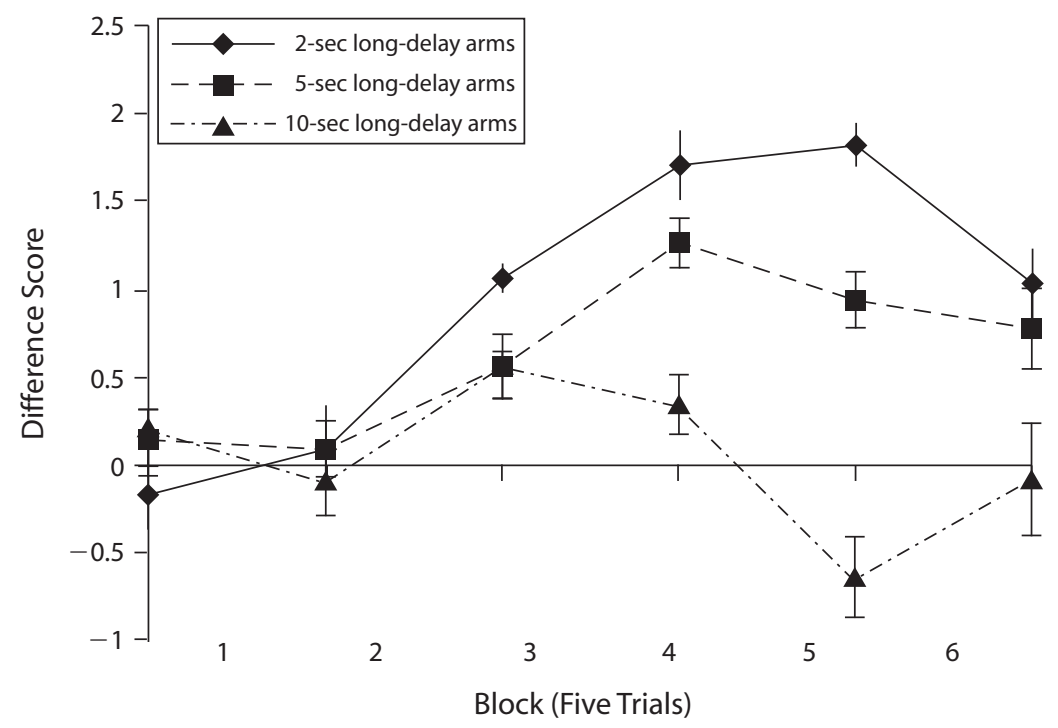

Figure 3. Difference scores among short- and long-delay mean ranks of entries for rats in the different long-delay conditions calculated for blocks of five trials in Experiment 1. Error bars represent standard errors of the mean.

two cases to actually prefer those arms and, in the third case, to demonstrate indifference.

Another possible explanation relates to the naturalism of the testing apparatus and procedures used in the present experiment as compared with the conditions of operant chambers and runway studies. Whishaw, Dringenberg, and Comery (1992) observed that when they were exposed, rats ate all food items significantly faster and exhibited significantly more scanning movements than when a means of cover was available. These results coincide with those of Phelps and Roberts (1989), who observed both that rats consumed food rewards faster when eating on radial maze arms than when eating on the central platform and that rats ran back to the central platform faster when they were carrying food items than when they were not carrying any food. These findings all seem to indicate an influence of perceived predation risk in foraging decisions, even in laboratory situations in which there is no actual threat of theft or predation, and suggest that rats treat the radial maze as a naturalistic environment.

It could be that the rats in the present experiment were also affected by environmental factors. The radial maze provided discrete foraging locations and a central, "safe" location. Given that this setup mirrors burrow systems, it may have induced more natural foraging than an operant chamber or a runway would have. In addition, this paradigm forced the rats to endure the delay to reward in an exposed position on the maze arms. It may be that the rats preferred the larger available food source up to a certain delay threshold - perhaps related in some way to the risk of predation per unit of delay exposed - after which point they became indifferent and potentially even switched their preferences. The open arms may have induced a natural fear of predation, making the rats more averse to the delay. This arm aversion could have affected the rats' perceptions of the trade-off between reward magnitude and risk of predation and may have prevented the 10 -sec long-delay group from showing a preference for the larger reward because the reward amount was not large enough to overcome the threat of predation; this aversion could have similarly reduced the strength of the preference exhibited by the 5 -sec long-delay group compared with that exhibited by the 2 -sec long-delay group.

\section{EXPERIMENT 2}

The design used in Experiment 1 was replicated in Experiment 2, but with additional modifications to the radial maze. The area in which the rats endured the delay to reward on each radial maze arm was fully enclosed with opaque poster board. The opaque walls and ceilings prevented the rats from seeing the exterior environment during the delay. Thus, the delay chambers again were associated with reward in all cases, but the views did not differ from arm to arm. If arms associated with a larger quantity of reward provided greater secondary reinforcement of arm choices in Experiment 1, that mechanism did not operate in Experiment 2. According to this reasoning, with differential secondary reinforcement eliminated, rats should show a tendency to choose the more immediately available reward before the delayed one.

To offer a contrasting theoretical point of view, rats might be more willing to endure the delay once the arms are transformed into tunnels, since tunnels are more similar to the rats' natural environment. It is known that rats do not readily venture into exposed environments, instead demonstrating a thigmotactic exploratory nature; they also construct multitunneled underground burrow structures. The addition of enclosures on the arms may thus reduce the rats' tendency to favor minimal delay on the arms even further than was the case when the arms were exposed. That is, rats might show an even greater prefer- 
ence for the arms containing the delayed large reward than they did in Experiment 1.

\section{Method}

Animals. The subjects were 15 (5 per group) experimentally naive, adult male hooded Long-Evans rats. At the start of testing, the animals were approximately 60 days old and weighed between 298 and $466 \mathrm{~g}(M=377 \mathrm{~g})$. They were maintained under the same conditions as were the rats used in Experiment 1.

Apparatus. The same radial arm maze that was used in Experiment 1 was used in Experiment 2, but the part of each arm in which the rats were delayed was transformed into an enclosed tunnel. Sections of $0.5-\mathrm{cm}$-thick black poster board, each section measuring $43.5 \mathrm{~cm}$ wide $\times 23 \mathrm{~cm}$ high, were attached to the maze between the guillotine frames on both sides of each of the radial maze arms. A third piece of poster board, used as a cover, was hinged on one side with black electrical tape, and it could be fastened to the other side using Velcro tabs. For Phase 2 of Experiment 2, one additional modification to the maze was used. Four pieces of Velcro were attached around the corners of the inner doorframes (adjacent to the central platform). Eight $12.6 \times 13.8 \mathrm{~cm}$ pieces of poster board were cut and attached to the lower half of the wooden guillotine frames. Four of these pieces of poster board were black and four were white, and they could be attached and removed from the doorframes with Velcro strips. Openings in these black and white covers, $5 \mathrm{~cm}$ wide $\times$ $5 \mathrm{~cm}$ high, acted as doorways for the rats to pass through.

Procedure. Training trials were carried out in the same manner as in Experiment 1, such that by the end of training all rats were reliably eating out of the food cups located at the ends of the radial maze arms and were habituated to the doors. Group assignment and testing replicated the between-subjects design used in Experiment 1. Rats were assigned randomly to one of three long-delay groups $(n=5)$. Trials were run in Phase 1 in the same manner as in Experiment 1, with enclosed tunnels on the arms of the maze and no distinguishing cues at the entrances to the arms.

It became apparent after 20 trials of training in Phase 1 that rats were not discriminating between short-delay and long-delay arms. In order to make the arm choices more discriminable, the black and white poster board doorways were attached to the arm entrances. For 3 randomly selected rats in each group, the long-delay arms were associated with the white doorways and the short-delay arms with the black doorways. The remaining 2 rats in each group were presented with the opposite associations. Rats continued to be trained at the rate of one trial per day for 20 trials in Phase 2.

\section{Results and Discussion}

Phase 1. The MREs into the short- and the long-delay arms during Phases 1 and 2 of Experiment 3 are shown for each delay group in blocks of five trials in Figure 4. During Phase 1, the 2-sec long-delay group showed an initial tendency over the first two blocks of trials to enter shortdelay arms sooner. This tendency disappeared in the final two blocks of Phase 1, for which the data indicate that the rats were indifferent to the choice between short- and longdelay arms. In contrast, across all four blocks of Phase 2, the 5-sec long-delay group showed an increasing tendency to enter the long-delay arms before the short-delay arms. The 5-sec long-delay group showed minimal, inconsistent separation in Phase 1. Like the rats in the 2-sec long-delay group, these rats exhibited a preference for the long-delay arms during Phase 2, which strengthened across the four blocks of trials to the point of being the strongest preference of any group. During Phase 1, the 10-sec long-delay group showed the largest separation, which was in the
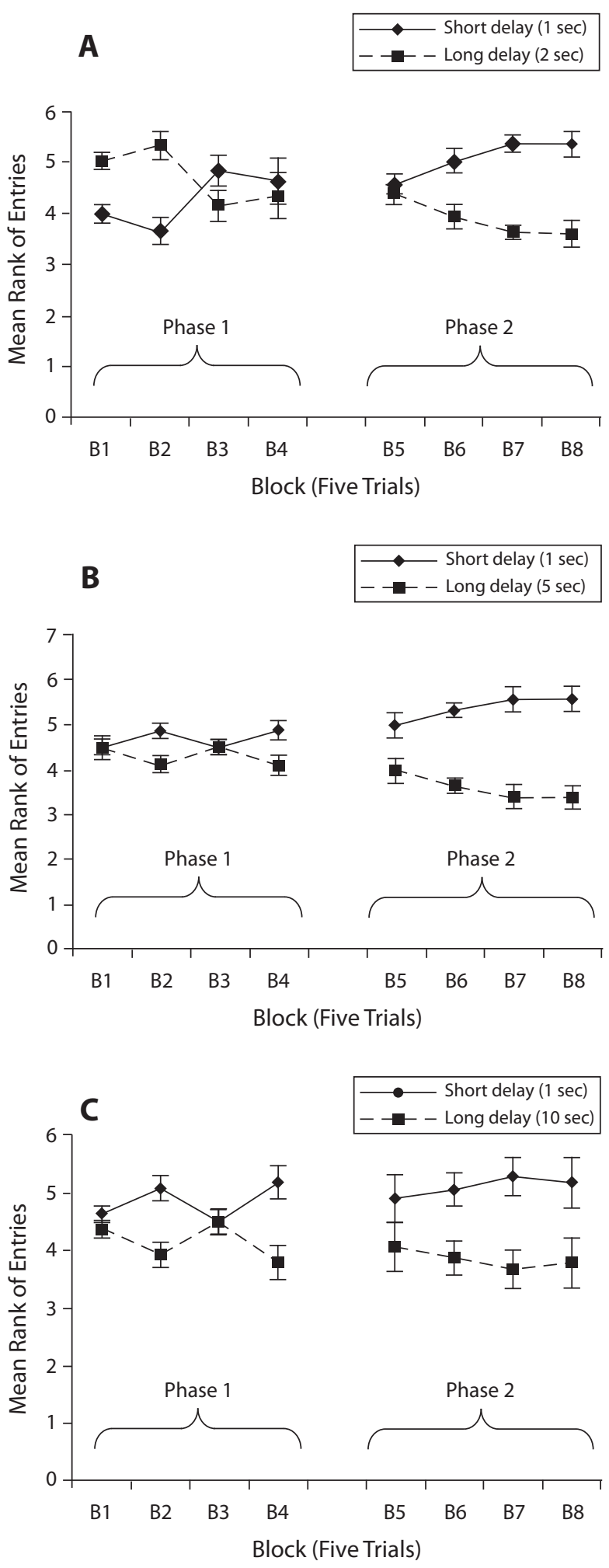

Figure 4. The mean ranks of entries into short- and long-delay arms for blocks of five trials during Phase 1 (no door cues) and Phase 2 (door cues) of Experiment 2. The short delay was always $1 \mathrm{sec}$, but the long delay varied across groups from $2 \mathrm{sec}(\mathrm{A})$ to $5 \mathrm{sec}(\mathrm{B})$ to $10 \mathrm{sec}(\mathrm{C})$. Error bars represent standard errors of the mean. 
direction of preferring the long-delay arms, but the preference was not demonstrated consistently across all four blocks. During Phase 2, the 10-sec long-delay group rats consistently entered long-delay arms sooner than they entered short-delay arms.

A set of three one-sample $t$ tests was used to test the difference scores between long- and short-delay MREs obtained in Phase 1 of Experiment 2 against zero (see Figure 5). The scores of the 2-sec long-delay group ( $M=$ $-.44, S D=.73)$, the 5 -sec long-delay group $(M=.78$, $S D=.95)$, and the $10-$ sec long-delay group $(M=.75$, $S D=.66)$ all failed to differ significantly from zero $[t(4)=1.33,1.83$, and 2.52 , respectively $]$.

A repeated measures, 3 (groups) $\times 4$ (blocks of five trials) ANOVA compared the difference scores in Phase 1 (see Figure 5 for a depiction of scores for each group in blocks of five trials during both phases). A significant interaction of group $\times$ block was observed $[F(6,36)=3.14, p<.014]$. Additionally, a significant main effect of block was obtained $[F(3,36)=2.94, p<.046]$. Finally, a group effect was found $[F(2,12)=3.94, p<.048]$. Post hoc tests of the group variable using pairwise comparisons with the LSD procedure indicated that the only significant difference was that the 2 -sec long-delay group differed from the 10-sec long-delay group. Further, post hoc comparisons employing the LSD procedure indicated that the Block 1 difference score was significantly different from that of Block 4 . No other significant differences between blocks were observed. Four followup one-way ANOVAs were run comparing group scores at each block separately, but no significant effects were found. However, the analysis of Block 2 approached significance $(p=.06)$, and post hoc tests using the LSD procedure from this analysis indicated that during Block 2 , the 10 -sec longdelay group had a significantly higher mean difference score $(M=1.17, S D=.97)$ than did the 2-sec long-delay group $(M=-.70, S D=1.62)$.
The results of Phase 1 indicate that none of the groups developed a preference for either set of arms. All groups were observed to show indifference to the two delay and magnitude alternatives. This finding supports the possibility that the rats required some form of differential immediate secondary reinforcement if they were to exhibit the choice patterns observed in Experiment 1. However, it could also be that the rats did not show a preference on the maze because they could not discriminate the entrances to the short- and long-delay arms. Rats typically employ extramaze cues (e.g., doors, objects, wall ornaments) in the testing room to navigate and remember spatial locations. Rats use intramaze cues (i.e., cues within the maze itself) to navigate if extramaze cues are not available or if only a low level of immediate reward can be obtained by relying on extramaze cues. In the present study, the installation of tunnels over the delay portion of the arms effectively blocked the rats' view of the extramaze cues. There were also no intramaze cues available. Thus, although rats still visited all of the arms, making relatively few repeat trips, they may not have been able to discriminate the various arms and thus to associate the differential contingencies with each arm, particularly since they were placed on the maze facing a different randomly selected arm on each trial.

Phase 2. The results of Phase 2 were analyzed with the same tests that were used for the data from Phase 1. Three one-sample $t$ tests were used to test the difference scores obtained in each group against zero. The mean difference scores for the 2-sec long-delay group $(M=1.18, S D=$ $.75)$ and the 5-sec long-delay group $(M=1.74, S D=.93)$ were significantly greater than zero $[t(4)=3.53, p=.024]$ and $[t(4)=4.19, p=.014]$, respectively. The mean difference score across all subjects in the 10-sec long-delay group $(M=1.26, S D=1.39)$ did not differ significantly from zero $[t(4)=2.03]$. However, one rat in this group,

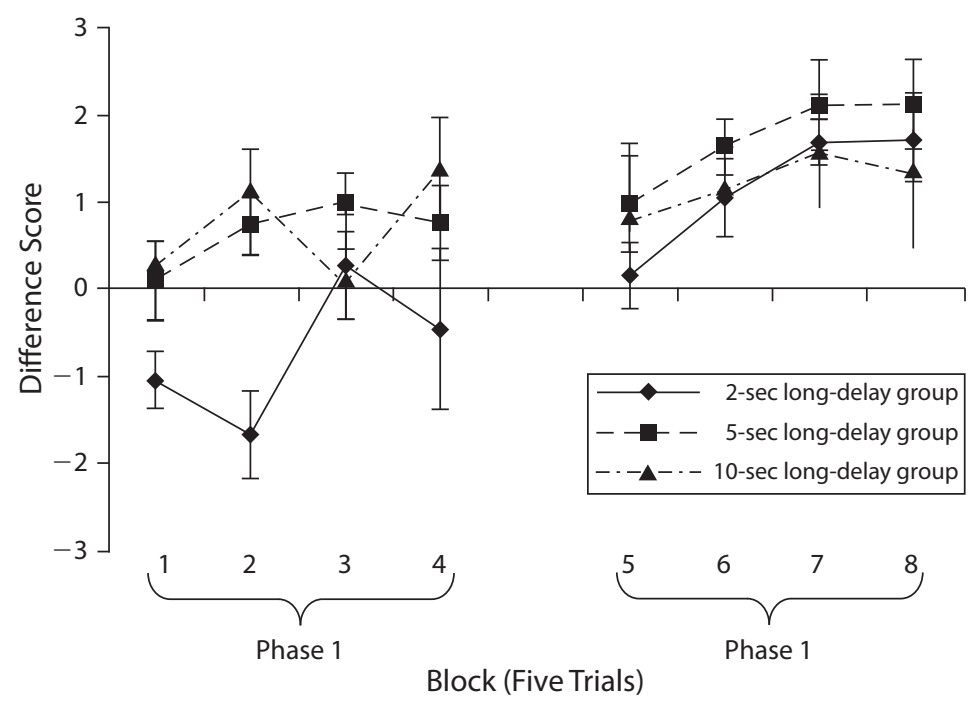

Figure 5. Difference scores among mean ranks of entries for short- and longdelay arms for rats in the different long-delay conditions calculated for blocks of five trials in Experiment 2. Black and white door cues were present in Phase 2 but not in Phase 1. Error bars represent standard errors of the mean. 
Rat 12 , showed a strong preference in the opposite direction from that of every other rat and behaved abnormally on the maze compared with the remaining 14 rats in the study. Rat 12 learned to lift the guillotine doors when the fishing line was taut. For this rat, the experimental task evolved into a task of running down the arms as fast as possible to get to the distal doors before the experimenter could put some slack in the fishing line. This rat appeared to be most concerned with getting beyond the doors as quickly as possible. Excluding the data from Rat 12, the analysis of the 10-sec long-delay group reveals that the difference score for this group of rats $(M=1.83, S D=$ $.64)$ is significantly larger than zero $[t(3)=5.73, p<.01]$ and is actually the highest mean difference of any group. All further analyses used data from only these 4 rats for the 10 -sec long-delay group.

The Phase 2 difference scores were analyzed using a 3 (groups) $\times 4$ (blocks of five trials) repeated measures ANOVA. In this analysis, no significant group effect or interaction of group $\times$ block was observed $\left(F_{\mathrm{S}}<1.0\right)$. There was a significant main effect of block $[F(3,36)=$ $6.52, p<.01]$. Post hoc testing using LSD pairwise comparisons illustrated that the Block 1 score differed from the scores of the other three blocks, and the Block 2 score differed from the Block 3 score. Four follow-up one-way ANOVAs were run comparing group scores at each block separately. As was the case in Phase 1, Phase 2 results did not demonstrate a significant effect of group during any block.

Finally, the results of Phases 1 and 2 were compared using a 3 (groups) $\times 2$ (phases) repeated measures ANOVA examining difference scores. The analysis did not indicate a significant interaction of group $\times$ phase $[F(2,12)=1.02, p=.39]$. There was not a significant main effect of group $[F(2,12)=2.09, p=.17]$. There was, however, a significant main effect of phase, indicating that the difference scores observed in Phase $2(M=1.39$, $S D=1.01)$ were significantly larger than those in Phase 1 $(M=.36, S D=.94)[F(1,12)=10.55, p=.01]$.

It can thus be observed that in Phase 2, rats provided with a set of intramaze cues that were correlated with delay and magnitude of reward showed a consistent trend in choice pattern. Preference emerged at the same rate in all three groups and was of the same magnitude. Rats entered arms offering five pellets delayed 2, 5, or $10 \mathrm{sec}$ before they entered arms offering one pellet delayed $1 \mathrm{sec}$. These findings are consistent with and extend the trends seen in Experiment 1. Since rats were able to demonstrate a preference after intramaze cues were installed on the radial maze in Phase 2, it can be concluded that the indifference exhibited by the rats in Phase 1 represented their inability to encode the exact spatial locations of the different reward magnitudes and delays due to a lack of orientation cues. It follows, then, that the preference for the arms offering larger, later rewards was not dependent on differential secondary reinforcement from environmental cues during the delay to reward.

We argue that the similarity of the testing situation to rats' natural burrow systems produced the preference for arms leading to the large reward, even though longer de- lays had to be endured. Delays for more preferred foods in a natural foraging situation may increase the risk of predation. In addition to being central-place foragers, rats are also thigmotactic in their locomotive behavior. They prefer complex environments that include many objects or walls to open environments; in the latter, they spend the majority of their time near any available wall structure (Arcis \& Desor, 2003; Genaro \& Schmidek, 2000). On the radial maze, rats do not spend time on the exposed arms and carry larger food items back to the central platform when possible (Phelps \& Roberts, 1989; Whishaw $\&$ Tomie, 1989). If the enclosed arms provided the rats with security from a perceived risk of predation, the differential delays spent in the arms may have been a minor factor in rats' arm choices, and amount of reward may have become the overriding consideration. The finding that the rats showed a more pronounced preference for the long-delay arms (which provided larger food rewards) when the arms were enclosed than when they were open suggests a sensitivity to environmental conditions that represents a trade-off between reward maximization and risk of predation.

Comparison of Experiment 1 and Experiment 2. The findings of Experiments 1 and 2 were next compared directly to shed further light on the proposed influence of reduced predation threat on the interaction of reward and delay effects. The difference scores were analyzed using a 3 (groups) $\times 4$ (blocks of five trials) $\times 2$ (experiments) repeated measures ANOVA. In this analysis, the group $\times$ block $\times$ experiment interaction, the group $\times$ block interaction, and the effect of group were not significant ( $p=.27, p=.78$, and $p=.51$, respectively). There was a significant block $\times$ experiment interaction $[F(3,69)=$ $3.84, p=.024]$ and an interaction of group $\times$ experiment $[F(2,23)=4.68, p=.02]$. In addition, there was a significant effect of block $[F(3,69)=3.15, p=.046]$, with post hoc tests indicating that Block 1 differed significantly from Blocks 2 and 3 . An effect of experiment was also found $[F(1,23)=9.12, p=.01]$, with Experiment 2 scores being larger than Experiment 1 differences.

Four follow-up one-way ANOVAs were run comparing Experiments 1 and 2 at each block separately. Across all groups, there were no significant differences in Blocks 1 and 2 ( $p=.67$ and $p=.31$, respectively). In Block 3 , the mean difference score in Experiment $2(M=1.98$, $S D=.93)$ was significantly larger than that of Experiment $1(M=.73, S D=1.3)[F(1,27)=8.89, p=.01]$. Similarly, during Block 4 , the mean difference score of Experiment $2(M=2.00, S D=1.10)$ was significantly larger than that of Experiment $1(M=.60, S D=1.32)$ $[F(1,27)=9.51, p=.01]$.

Three repeated measures, 4 (blocks) $\times 2$ (experiments) ANOVAs were run, one for each delay group. For the 2 -sec long-delay group, there was a significant block $\times$ experiment interaction $[F(3,24)=3.52, p=.03]$. There was also a significant effect of block, indicating that Block 1 differed from the other three blocks. For the 5-sec long-delay group, there was a significant effect of block $[F(3,24)=3.46, p=.03]$. Block 1 differed from Blocks 2 and 3 . For the 10 -sec long-delay group, there was a sig- 
nificant effect of experiment $[F(1,7)=20.89, p=.003]$. Difference scores for Experiment 2 were larger than those documented for Experiment 1.

Three follow-up one-way ANOVAs compared Experiments 1 and 2 for each group at each of the four, five-trial blocks. No significant differences were found between Experiments 1 and 2 for the 2- and 5-sec long-delay groups at any block. For the 10-sec long-delay group, however, the difference scores found in Experiment 2 during Block $2(M=$ $1.68, S D=.80)$ and Block $3(M=2.10, S D=1.14)$ were significantly larger than those found in Experiment 1 during Block $2(M=.36, S D=.52)$ and Block $3(M=-.62$, $S D=.99),[F(1,7)=8.91, p=.02$ and $F(1,7)=14.66, p=$ .01 , respectively]. The difference between the Experiment 2 scores $(M=2.13, S D=1.36)$ and Experiment 1 scores $(M=-.06, S D=1.54)$ during Block 4 approached but did not reach significance $[F(1,7)=4.91, p=.06]$.

A group $\times$ experiment ANOVA was run to compare difference scores across all trials. A significant group $\times$ experiment interaction was observed $[F(1,23)=4.67, p=$ $.02]$. As well, there was a significant effect of experiment $[F(1,23)=9.11, p=.006]$, with Experiment 2 difference scores being larger than Experiment 1 difference scores. An independent samples $t$ test comparing the 2-sec longdelay group's difference scores from Experiment $1(M=$ $1.42, S D=.70$ and $M=1.18, S D=.75)$ indicated no difference $[t(8)=.54, p=.61]$. For the 5 -sec long-delay group, there was no difference $[t(8)=1.75, p=.12]$, but the trend was in the direction of the Experiment 2 scores $(M=1.74, S D=.93)$ being larger than the Experiment 1 scores $(M=.83, S D=.69)$. In the case of the $10-\mathrm{sec}$ long-delay group, the Experiment 2 difference scores $(M=1.83, S D=.64)$ were significantly larger than the Experiment 1 difference scores $(M=0.07, S D=.52)$.

In sum, the difference score data indicate that when there were differences, it was overwhelmingly the case that the rats in Experiment 2 had larger difference scores than did the rats in Experiment 1 and that this pattern was displayed earlier in Experiment 2. This means that across all groups, Experiment 2 animals were more likely to enter the long-delay, large-reward arms earlier in a trial than were the animals from Experiment 1 . These findings support the idea that the rats in Experiment 2 chose larger rewards at longer delays earlier than they chose smaller rewards at shorter delays because the fear of predation was reduced.

An alternative account of the differences between Experiments 1 and 2 was offered by reviewers of an earlier version of this manuscript, who suggested that the introduction of tunnels in Experiment 2 caused the rats to navigate the maze more slowly than they did in Experiment 1, which thus added a constant to the delays to reward. In operant chamber studies (Green \& Estle, 2003), the addition of a constant to short and long delays to reward shifted preferences toward the longer delay and larger reward arms. In the present study, the total time to complete the maze was recorded for both Experiments 1 and 2. These times were averaged over the last three trials of the final block of trials for each experiment, and the mean scores are shown in Table 1. These times were corrected
Table 1

Corrected Mean Time and Standard Errors of the Mean (in Seconds) for Total Time Rats Spent on the Maze in Each Long-Delay Condition

\begin{tabular}{lrrrrr}
\hline & \multicolumn{2}{c}{ Experiment 1} & & \multicolumn{2}{c}{ Experiment 2 } \\
\cline { 2 - 3 } \cline { 5 - 6 } Group & $M$ & \multicolumn{1}{c}{$S E$} & & $M$ & $S E$ \\
\hline $2 \mathrm{sec}$ & 133.33 & 25.57 & & 147.53 & 13.37 \\
$5 \mathrm{sec}$ & 129.86 & 9.81 & & 135.46 & 51.21 \\
$10 \mathrm{sec}$ & 133.46 & 19.42 & & 154.55 & 42.07 \\
\hline
\end{tabular}

by subtracting from each score the time a rat was delayed in short- and long-delay alleys.

The mean times are slightly higher in Experiment 2 than in Experiment 1 and appear to differ little among groups. The corrected total time required by the rats to complete a trial was examined in a 3 (groups) $\times 2$ (experiments) ANOVA. The analysis showed that there was no significant effect of group, experiment, or group $\times$ experiment interaction (all $F_{\mathrm{s}}<1.0$ ). These results indicate that rats completed the tasks in the same amount of time both when the arms were exposed and when they were enclosed.

\section{EXPERIMENT 3}

In Experiment 3, an alternative account for the findings of Experiment 2 was tested. This account suggests that choices were influenced more by characteristics of the maze environment than by the specific reward contingencies employed. Recall that choice of the larger, more delayed reward arms in Experiment 2 also led animals to spend more time in an enclosed dark space. The preference for the larger, later rewards in all delay conditions shown in Experiment 2 might have reflected rats' characteristic thigmotactic nature. If access to the delay chamber was in itself rewarding, rats may have chosen the arms with longer delays because they preferred a long stay in the alley over a short stay in the alley, not because the long delay condition led to larger rewards. The present experiment tested this hypothesis by providing rats with differential delays across sets of arms but with the same magnitude of reward on all arms. Only one group of rats was tested, with delays of 1 and $10 \mathrm{sec}$. If rats' preference for the arms with longer delays and larger rewards in Experiment 2 was based solely on a preference for a long stay in the delay alley, then rats should have preferred the 10 -sec arms to the 1-sec arms in Experiment 3.

\section{Method}

Animals. Five experimentally naive, adult male hooded LongEvans rats were used. At the start of testing, the animals were approximately 90 days old and weighed between 358 and $387 \mathrm{~g}(M=$ $376.8 \mathrm{~g})$. The rats were maintained in the same way as the rats used in Experiments 1 and 2.

Apparatus. The radial arm maze used in Experiment 2 was used, with the tunnel modifications maintained and the black and white door cues employed from the start of testing.

Procedure. The animals were trained to eat sucrose pellets out of the food cups reliably and were habituated to the guillotine doors. Response criteria and choice conditions were consistent with those used in the previous experiments. The 5 rats were tested for 20 days, with one trial on each day. Each of the eight arms on the maze con- 
tained one sucrose pellet on each trial. For each rat, four arms were chosen randomly to be 1-sec delay arms, and the other four arms served as 10 -sec delay arms. For 2 rats, white doors always led to 1 -sec delay alleys, and black doors always led to 10-sec delay alleys; the associations between door cues and delay were reversed for the other 3 rats. Throughout testing, rats could choose freely among the eight arms until all eight had been entered on a trial.

\section{Results}

Difference scores were obtained by subtracting each rat's individual MRE into the 10-sec delay arms from its MRE into the 1-sec delay arms. A positive number indicates that the 10-sec delay arms were entered before the 1 -sec delay arms, whereas a negative number indicates that the 1-sec delay arms were entered earlier. Four onesample $t$ tests were used to determine whether the difference score for each block of Experiment 4 differed from zero. The mean difference score across all rats in Block 1 $(M=-0.37, S D=.85)$ and Block $2(M=0.80, S D=$ 1.10) were not significantly different from chance $[t<$ 1.0 and $t(4)=1.62$, respectively]. The average difference score in Block $3(M=1.94, S D=0.70)$ was significantly lower than a score of zero $[t(4)=6.21, p<.01]$. Finally, in Block 4, the trend observed in Block 3 continued, and the difference score was significantly lower than zero $(M=2.48, S D=1.0)[t(4)=5.55, p<.01]$.

An ANOVA revealed a significant effect of block $[F(3,12)=10.98, p<.001]$. Post hoc pairwise comparisons using the LSD adjustment showed that both the Block 1 and Block 2 difference scores were significantly smaller than those of Blocks 3 and 4. No further pairwise differences were found.

\section{Discussion}

The results of Experiment 3 clearly indicate that rats came to prefer the short-delay arms over the long-delay arms. Since there was no difference in reward magnitude among the arms, rats clearly preferred the shorter delay to reward and, by the same token, did not prefer the cues that signaled longer stays in the alleys. It can therefore be concluded that the rats' preference for the more delayed but larger magnitude rewards in Experiment 2 was not just the product of a preference for an enclosed delay alley.

\section{GENERAL DISCUSSION}

The results of Experiment 1 show that rats choosing between one pellet delayed for $1 \mathrm{sec}$ and five pellets delayed for either 2 or $5 \mathrm{sec}$ preferred the larger, later rewards. However, the learning curves of the two groups varied in important ways: The 2-sec long-delay group showed more rapid acquisition and a stronger preference relative to the 5 -sec long-delay group. Rats in the 10-sec long-delay group were indifferent to the choice between one pellet delayed for $1 \mathrm{sec}$ and pellets delayed for $10 \mathrm{sec}$. In Experiment 2 , three groups of rats showed the same degree of preference for long-delay, large-reward arms when the arms were made into tunnels. Rats visited arms containing five pellets delayed for 2, 5, or $10 \mathrm{sec}$ before they visited arms offering 1 pellet delayed for $1 \mathrm{sec}$. These findings are consistent with the trends identified in Experiment 1 and both extend and strengthen them. Rats were clearly more influenced by amount of reward than by delay to reward when the delay was spent in an enclosed space.

Overall, the results of this series of experiments indicate that the choice of smaller, sooner rewards over larger, later rewards by rats may be partially determined by the experimental situation. The present experiments showed that in a radial maze set up to allow rats to forage through tunnels that may to some degree have mimicked a burrow system, rats chose to visit the arms of the maze that offered larger but more delayed food sources before they visited arms offering smaller but more immediate food sources. The tendency to show a preference for a larger reward rather than for a shorter delay to a smaller reward appeared as an initial and consistent preference rather than as a reduction in the baseline rate of choice. These findings suggest that factors governing optimal foraging for animals must be considered in studies of choices among delayed rewards that vary in magnitude, as well as in studies of cognitive processes suggested to account for impulsive behavior, rapid discounting of delayed rewards, and short time horizons.

We suggest that the covers installed on the maze arms in Experiment 2 functioned to reduce fear of predation. If rats felt more protected in the enclosed arms during a delay, preferences for larger rewards could be expressed unaffected by predation risk. The reduced preference for the long-delay arms of the 2-sec and 5-sec long-delay groups and the indifference shown by the 10-sec longdelay group in Experiment 1 may have resulted from the influence of the threat of predation. Rats seem to naturally react to subjective threat of predation cues in radial maze laboratory work (Roberts, 1992), and this factor would have been more salient and uncertain with the exposed arms used in Experiment 1 than with the enclosed arms used in Experiment 2. The indication is that rats may be able to recognize the energetic value of a food source in terms of food density but that they also recognize the costs of vigilance requirements and will more readily enter richer patches when predation threats are not substantially high. This argument nicely explains the discrepant findings of Experiments 1 and 2.

In some respects, the findings reported here for rats on the radial maze may seem puzzling when compared with results from rats tested in operant chambers. Rats tested on the maze showed preferences for large, delayed rewards or indifference between large, delayed rewards and small, immediate rewards on the open maze and more complete preference for large, delayed rewards on the maze with enclosed alleys. We attributed this increased preference for large, delayed rewards on the enclosed-alley maze to reduction of fear of predation. However, in an enclosed operant chamber, rats preferred small, immediate rewards (Green \& Estle, 2003; Tobin, Chelonis, \& Logue, 1993), and it may be argued that fear of predation should have been minimal in such an enclosed setting. A possible integration of these findings may arise from the finding that rats in an operant chamber increased their preference for the larger, 
delayed alternative as a constant delay was added to the delivery of both the small and the large reinforcers (Green \& Estle, 2003). In the present study, the short delay on the radial maze lasted $1 \mathrm{sec}$ - the door at the end of the alley was opened $1 \mathrm{sec}$ after a rat entered the alley. However, rats took some extra time to go through the door and proceed to the food cup at the end of the alley. The 1-sec delay plus this extra traveling time may have been sufficiently long to shift preference away from the short-delay, smallreward alternative, as did the addition of a constant delay in the operant chamber. However, this possibility does not account for the difference between Experiments 1 and 2 . Although rats took the same amount of time to go through the maze in both experiments, the addition of enclosed alleys in Experiment 2 strongly increased rats' preference for the long-delay, large-reward arms.

The persistent conclusion that the process of choosing between smaller, more immediate rewards and larger, more distant ones differs among organisms in direct relation to the delays involved in the choice alternatives leads to the hypothesis of quantitative variability in choice preferences across species (Green \& Estle, 2003). Rachlin (1974) suggested an interpretation of the discrepancy between human and animal behavior that complements this hypothesis. He proposed that the difference does not lie in the source of the factors that control behavior (external cues in the case of rats, internal direction in the case of humans). Instead, Rachlin argued that human cognition may simply be more sensitive to outcomes occurring at a further remove from the contingent behavior. Rats may not be as capable of recognizing cause and effect relations between events over as large a time span as humans are. This seems to be a fundamentally quantitative description of the difference between animal and human behavior that does not appeal to different processes.

Factors other than perception of relationships over time, such as risk of predation and metabolic needs, may also be incorporated into foraging decisions, however. The findings reported here suggest that optimal foraging decisions made by rats among choices that offer different time delays to food and different amounts of food are heavily influenced by contextual factors concerned with risk of predation. When the appearance of risk is minimized, rats behave like foragers favoring the largest patches of food in the environment, not like impulsive organisms seeking out the fastest available food source. Presumably, adding covers to the maze arms did not increase rats' ability to anticipate more distant future events. An alternative is that the rats could readily anticipate those events but took advantage of this foresight only when they could do so safely.

\section{AUTHOR NOTE}

Support for this research was provided by a discovery grant to W.A.R. from the Natural Sciences and Engineering Research Council of Canada. Correspondence concerning this article should be addressed to M. C. Feeney or W. A. Roberts, Department of Psychology, University of Western Ontario, London, ON, N6A 5C2 Canada (e-mail: mcfeeney@uwo .ca or roberts@uwo.ca).

Note-This article was accepted by the previous editorial team, when Shepard Siegel was Editor.

\section{REFERENCES}

ARCIS, V., \& DESOR, D. (2003). Influence of environmental structure and food availability on the foraging behavior of the laboratory rat. Behavioural Processes, 60, 191-198.

Chelonis, J. J., Logue, A. W., Sheehy, R., \& Mao, J. (1998). Effects of response effort on self-control in rats. Animal Learning \& Behavior, 26, 408-415.

Clayton, K. N. (1964). T-maze learning as a joint function of the reward magnitude for the alternatives. Journal of Comparative \& Physiological Psychology, 58, 333-338.

DAVENPORT, J. W. (1962). The interaction of magnitude and delay of reinforcement in spatial discrimination. Journal of Comparative \& Physiological Psychology, 55, 267-273.

Eisenberger, R., Masterson, F. A., \& Lowman, K. (1982). Effects of previous delay of reward, generalized effort, and deprivation on impulsiveness. Learning \& Motivation, 13, 378-389.

Eisenberger, R., Weier, F., Masterson, F. A., \& Theis, L. Y. (1989). Fixed-ratio schedules increase generalized self-control: Preference for large rewards despite high effort or punishment. Journal of Experimental Psychology: Animal Behavior Processes, 15, 383-392.

Flaherty, C. F., \& Checke, S. (1982). Anticipation of incentive gain. Animal Learning \& Behavior, 10, 177-182.

ForZANo, L. B., \& Logue, A. W. (1994). Self-control in adult humans: Comparison of qualitatively different reinforcers. Learning \& Motivation, 25, 65-82.

Forzano, L. B., \& Logue, A. W. (1995). Self-control and impulsiveness in children and adults: Effects of food preferences. Journal of the Experimental Analysis of Behavior, 64, 33-46.

GenARo, G., \& SchmideK, W. R. (2000). Exploratory activity of rats in three different environments. Ethology, 106, 849-859.

Green, L., \& Estle, S. J. (2003). Preference reversal with food and water reinforcers in rats. Journal of the Experimental Analysis of Behavior, 70, 233-242.

Green, L., \& Meyerson, J. (2004). A discounting framework for choice with delayed and probabilistic rewards. Psychological Bulletin, 130, 769-792.

GRICE, G. R. (1948). The relation of secondary reinforcement to delayed reward in visual discrimination learning. Journal of Experimental Psychology, 38, 1-16.

Hull, C. L. (1943). Principles of behavior. New York: AppletonCentury-Crofts.

Hulse, S. H., \& O'Leary, D. K. (1982). Serial pattern learning: Teaching an alphabet to rats. Journal of Experimental Psychology: Animal Behavior Processes, 8, 260-273.

JACKSON, T. P. (2001). Factors influencing food collection behaviour of Brants' whistling rat (Parotomys brantsii): A central place forager. Journal of Zoology, 255, 15-23.

KING, G. R., \& LoGUE, A. W. (1987). Choice in a self-control paradigm with human subjects: Effects of changeover delay duration. Learning \& Motivation, 18, 421-438.

KirK, J. M., \& LoGUE, A. W. (1997). Effects of deprivation level on humans' self-control for food reinforcers. Appetite, 28, 215-226.

Lair, H., Kramer, D. L., \& Giraldeau, L. A. (1994). Interference competition in central place foragers: The effect of imposed waiting on patch-use decisions of eastern chipmunks, Tamias striatus. Behavioral Ecology, 5, 237-244.

LETT, B. T. (1973). Delayed reward learning: Disproof of the traditional theory. Learning \& Motivation, 4, 237-246.

Lieberman, D. A., McIntosh, D. C., \& Thomas, G. V. (1979). Learning when reward is delayed: A marking hypothesis. Journal of Experimental Psychology: Animal Behavior Processes, 5, 224-242.

Lima, S. L. (1985). Maximizing feeding efficiency and minimizing time exposed to predators: A trade-off in the black-capped chickadee. Oecologia, 66, 60-67.

Lima, S. L., Valone, T. J., \& Caraco, T. (1985). Foraging-efficiencypredation-risk trade-off in the grey squirrel. Animal Behaviour, 33, $155-165$.

Logan, F. (1965). Decision making by rats: Delay versus amount of reward. Journal of Comparative \& Physiological Psychology, 59, $1-12$.

Logue, A. W. (1988). Research on self-control: An integrating framework. Behavioral \& Brain Sciences, 11, 665-709. 
Logue, A. W., Peña-Correal, T. E., Rodriguez, M. L., \& Kabela, E. (1986). Self-control in adult humans: Variation in positive reinforcer amount and delay. Journal of the Experimental Analysis of Behavior, 46, 159-173.

Mazur, J. E., \& Logue, A. W. (1978). Choice in a "self-control" paradigm: Effects of a fading procedure. Journal of the Experimental Analysis of Behavior, 30, 11-17.

Meltzer, D., \& Brahlek, J. A. (1968). Quantity of reinforcement and fixed-interval performance. Psychonomic Science, 12, 207-208.

Neider, L., Cagnin, M., \& Parisi, L. (1982). Burrowing and feeding behavior in the rat. Animal Behaviour, 30, 837-844.

Olton, D. S., \& SAmuelson, R. J. (1976). Remembrance of places passed: Spatial memory in rats. Journal of Experimental Psychology: Animal Behavior Processes, 2, 97-116.

Phelps, M. T., \& Roberts, W. A. (1989). Central place foraging by Rattus norvegicus on a radial maze. Journal of Comparative Psychology, 103, 326-338.

Pisano, R. G., \& Storer, T. I. (1948). Burrows and feeding of the Norway rat. Journal of Mammalogy, 29, 374-383.

RaCHLIN, H. (1974). Self-control. Behaviorism, 2, 94-107.

RACHLIN, H., \& GREEN, L. (1972). Commitment, choice and self-control. Journal of the Experimental Analysis of Behavior, 17, 15-22.

RoberTs, W. A. (1969). Resistance to extinction following partial and consistent reinforcement with varying magnitudes of reward. Journal of Comparative \& Physiological Psychology, 67, 395-400.

RoberTs, W. A. (1992). Foraging by rats on a radial maze: Learning, memory, and decision rules. In I. Gormezano \& E. A. Wasserman (Eds.), Learning and memory: The behavioral and biological substrates (pp. 7-23). Hillsdale, NJ: Erlbaum.

Roberts, W. A., Phelps, M. T., \& Schacter, G. B. (1992). Stimulus control of central place foraging on the radial maze. In W. K. Honig \& J. G. Fetterman (Eds.), Cognitive aspects of stimulus control (pp. 135-153). Hillsdale, NJ: Erlbaum.

SPENCE, K. W. (1947). The role of secondary reinforcement in delayed reward learning. Psychological Review, 54, 1-8.

SPENCE, K. W. (1956). Behavior theory and conditioning. New Haven: Yale University Press.

Stephens, D. W., \& Krebs, J. R. (1986). Foraging theory. Princeton, NJ: Princeton University Press.

Tobin, H., Chelonis, J. J., \& Logue, A. W. (1993). Choice in selfcontrol paradigms using rats. Psychological Record, 43, 441-453.

Whishaw, I. Q., Dringenberg, H. C., \& Comery, T. A. (1992). Rats (Rattus norvegicus) modulate eating speed and vigilance to optimize food consumption: Effects of cover, circadian rhythm, food deprivation, and individual differences. Journal of Comparative Psychology, 106, 411-419.

Whishaw, I. Q., \& Tomie, J.-A. (1989). Food-pellet size modifies the hoarding behavior of foraging rats. Psychobiology, 17, 93-101.

(Manuscript received December 4, 2006; revision accepted for publication August 29, 2007.) 